PROKLA-Redaktion

\title{
Editorial: S(t)andOrt Berlin
}

Daß die PROKLA nach dem Heft StadTRaum (PROKLA 109, Dezember 1997) nun dem "neuen Berlin" ein weiteres Stadtheft widmet, ist keineswegs einem Lokalpatriotismus der Redaktion geschuldet. Wenn Berlins Innensenator Schönbohm mahnt: "Berlin muß endlich begreifen, daß es nicht nur sich selber gehört," heißt dies ja wohl, daß auch unsere LeserInnen im "Rest" der Bundesrepublik ihre Ansprüche und Interessen an Berlin haben. Berlin, so Schönbohm, "repräsentiert die Mitte Deutschlands in der Weltöffentlichkeit", und an der wachsenden internationalen Literatur zu Berlin könnte man in der Tat ablesen, daß nicht nur das deutsche Interesse an dieser Stadt explodiert ist. Die außergewöhnlichen Bedingungen, die mit dem Mauerfall für politische, wirtschaftliche und räumliche Entwicklungen eröffnet wurden, machten Berlin zu einem Experimentierfeld "am Ende der Geschichte", wo sich kapitalistische Träume und stadtentwicklungspolitische Hoffnungen im Scheinwerferlicht internationalen Interesses entfalten - oder in den märkischen Sand setzen ließen.

Um die 1989/90 geweckten Träume von der prosperierenden Metropole von Weltformat, der europäischen Dienstleistungsmetropole und der Ost-WestDrehscheibe zu verwirklichen, setzten die regierenden Politiker vor allem auf internationale Projektentwickler und ihre Financiers, machten die Rationalität der umworbenen Investoren zur Grundlage ihrer Entscheidungen - zur Not auch unter Umgehung oder entsprechender Abänderung lokaler Entscheidungsstrukturen zum Zwecke beschleunigter Handlungsfähigkeit. So entstand mit dem »Koordinierungsausschuß für innerstädtische Investitionen « ein in keiner Verfassung vorgesehenes, von keinem Parlament zu kontrollierendes Gremium, das von der Öffentlichkeit weitgehend unbemerkt in den Jahren 1991-93 Grundsatzentscheidungen über die Gestaltung des "Zentralen Bereichs" von Berlin getroffen hat, an deren ökonomisch und stadtpolitisch höchst problematischen Auswirkungen die Stadt noch einige Jahrzehnte zu tragen haben wird. Die beschleunigten Investitionsentscheidungen ließen eine ganze Riege von überdimensionierten Projekten entstehen, die - wie sich schnell zeigte zu einem Großteil am Berliner Markt vorbei geplant waren (vergl. den Beitrag von Karin Lenhardt).

Liest man heute die Anfang der 90er Jahre entstandenen Prognosen zur künftigen wirtschaftlichen Entwicklung Berlins, so entsteht der Eindruck, es muß sich dabei um eine andere Stadt gehandelt haben. Statt des Zustroms hochqua- 
lifizierter Neuberliner, ständig wachsender Immobiliennachfrage und einer pulsierenden Dienstleistungsmetropole sieht sich Berlin heute mit der Abwanderung der besserverdienenden Familien ins Umland, einer immer weiter steigenden Arbeitslosigkeit und einer rapide zunehmenden Verschuldung der öffentlichen Hand konfrontiert. Daß mit der bloßen Fortsetzung einer Subventionierung von Investitionen und der Förderung von Existenzgründungen hier keine Veränderung eingeleitet werden kann, macht der Beitrag von Günter Seiler deutlich.

Wirkungslos blieb die Berliner Politik allerdings nicht: Die Finanzkrise des Senats, die sich sowohl dem Abbau der Berlin-Förderung als auch den großzügigen (sowohl in Berlin als auch in Bonn beschlossenen) Steuergeschenken an Investoren verdankt, führte immerhin zu einer radikalen Schrumpfkur für die Kultur- und Bildungseinrichtungen. Allein die Ausgaben für die drei Hochschulen der Stadt wurden innerhalb kürzester Zeit um ein Drittel reduziert. Was in den Verlautbarungen des Senats noch immer gerne als besonderer Berliner »Standortfaktor « gepriesen wird die Hochschul- und Wissenschaftslandschaft -, ist von demselben Senat in weiten Teilen bereits zur Abwicklung freigegeben worden.

Im $\mathrm{Ma}$ wie der Optimismus der frühen 90er Jahre auf allerlei Hindernisse stieß, und die ökonomischen Rahmendaten immer schlechter wurden, zogen die an der Gestaltung des "neuen Berlin" beteiligten Akteure eine weitere wichtige Lehre: der Traum von der prosperierenden

Dienstleistungsmetrople läßt sich nur verwirklichen, wenn der Standort Berlin nicht weiter »schlechtgeredet « wird. Viel Energie, Arbeitszeit und
Ressourcen gingen folglich in die Produktion und Verbreitung von Hochglanzbroschüren, eine Phalanx von Publikationen, sowie in unzählige Veranstaltungen, Ausstellungen, Wettbewerbe und die Inszenierung stadtpolitischer Debatten, in denen viel um Architektur und wenig um Nutzungen und soziale Ansprüche gerungen wurde. »Imagepolitik « wird in Berlin großgeschrieben. Dabei geht es nicht nur um belanglose Bilder, die einer internationalen Öffentlichkeit von Berlin vermittelt werden sollen. Wie Susanne Heeg in ihrem Beitrag zeigt, spielen die entsprechenden Bilder und Diskurse eine erhebliche Rolle bei der Neuformulierung einer neoliberal orientierten städtischen Politik.

Während die Projektentwickler mit Bürobauten, für die es keinen Bedarf gibt, Steuergeschenke realisieren, macht der neue Sozialatlas (vgl. taz vom 31.1.98) sowie ein von Stadtentwicklungssenator Strieder in Auftrag gegebenes Gutachten "Soziale Stadtentwicklung" (vgl. taz vom 27.1.98) deutlich, daß das neue Berlin von einer immer stärkeren sozialen Polarisierung gekennzeichnet ist und immer dunklere Schattenseiten aufweist. Das soeben vorgelegte Gutachten zur sozialen Stadtentwicklung identifiziert beispielsweise sechs Armutskieze, in denen schlechte Wohnausstattung und Überbelegung, illegale Unterbringung von Bürgerkriegsflüchtlingen in privaten Wohnpensionen, hohe Fluktuation der Bewohner, Verwahrlosung des Wohnumfelds, Drogenszenen, hohe Arbeitslosigkeit und Sozialhilfedichte die vorherrschenden Merkmale sind.

Von den wachsenden gesellschaftlichen Spannungen blieben auch die Jugendkulturen nicht verschont. Die Rede von »Gangs« ist nicht nur bei einer sensa- 
tionsheischenden Presse, sondern auch bei den Jugendlichen selbst beliebt. Daß Jugendgangs in Berlin aber etwas ganz anderes darstellen als ihre medial vermittelten Vorbilder in amerikanischen Städten, weil sie trotz Kleinkriminalität und gewaltsamen Auseinandersetzungen eben nicht in eine umfassendere kriminelle Ökonomie integriert sind, macht Uli Jähner deutlich.

Auf die zunehmende Verarmung und Obdachlosigkeit mit all ihren nicht zu den Vorstellungen von einer properen Metropole passenden Begleiterscheinungen wird vom offiziellen Berlin zunehmend repressiv reagiert, vom $\gg \mathrm{Fi}-$ xerjogging «, der polizeilichen Vertreibung der Drogenszene von einem Treffpunkt zum nächsten, bis zur »Verbringung « von das Stadtbild störenden Obdachlosen in den Grunewald. Erst recht trifft diese Politik solche Gruppen, die wie Hausbesetzer oder die Bewohner von »Wagenburgen« alternative Lebens- und Wohnvorstellungen entwickeln. $\mathrm{Da}$ es sich dabei nicht um marginale Bereiche großstädtischer Politik handelt, sondern um die Definition städtischen Raums zeigen Jens Sambale und Dominik Veith in ihrem Beitrag.

Die zunehmende Privatisierung von Sicherheitsdienstleistungen, mit der sich Volker Eick auseinandersetzt, läßt nicht nur das staatliche Gewaltmonopol, zumindest was die innerstädtischen Bereiche angeht, in Richtung einer »privatepublic-partnership « tendieren; sie ist auch von einschneidenden Veränderungen des öffentlichen Raumes begleitet, wovon die Privatisierung des »Los Angeles Platzes « in der Berliner City-West nur das augenfälligste Beispiel ist.

$\mathrm{Da}$ sich dieses Heft vor allem mit den negativen Seiten Berlins beschäftigt, hat seinen guten Grund. Jubelliteratur, die die Literaturlandschaft im In- und Aus- land beherrscht, gibt es schon in Hülle und Fülle. ${ }^{1}$ Nachdem es mit der von vielen politischen Akteuren erträumten boomenden Dienstleistungsmetropole nichts werden will, klammert sich die politische Klasse der Stadt jetzt an die einzig verbleibende Hoffnung den unangenehmen Realitäten zu entgehen, den Regierungsumzug. Es ist also höchste Zeit, den "Standort" Berlin "schlechtzureden", wenn es denn doch einmal zu so etwas wie einer öffentlich und demokratisch geführten Auseinandersetzung darüber kommen soll, welche $\mathrm{Zu}$ kunft für diese Stadt angestrebt wird.

$$
* * *
$$

Außerhalb des Schwerpunkts nehmen wir mit dem Artikel von Ekkehard Hein eine Debatte über Marx und die moderne ökonomische Theorie auf, die in einem der nächsten Hefte mit einem Beitrag von Klaus Schabacker fortgesetzt werden wird.

1 Siehe z.B. Werner Süß (Hg.), Hauptstadt Berlin. Band 1: Nationale Hauptstadt. Europäische Metropole, Bd. 2: Berlin im vereinten Deutschland, Bd. 3: Metropole im Umbruch. Berlin Verlag 1994-1996; Anthony Grafton, "Hello to Berlin," New York Review of Books, 14. August 1997; P. Goldberger, "Berlin must look like Berlin," New York Times Magazine, 5. Februar 1995. 